http://revistaurbanismo.uchile.cl

\title{
Medio ambiente urbano: Una mirada desde la historia de las ideas científicas y las profesiones de la ciudad. Buenos Aires 1850-1915.
}

Verónica Paiva

\section{Filiación}

Magíster en Gestión Ambiental, doctora en Ciencias Sociales y docente de FADU-UBA

\section{Resumen}

Desde la década de 1960 en adelante, el medio ambiente se ha convertido en un tema prioritario en el nivel mundial. En lo que a estudios históricos se refiere la mayor parte de las investigaciones sobre historia ambiental han sido producidas desde la perspectiva ecológica, es decir de una mirada que toma conceptos actuales del debate ambiental (relación sociedad-naturaleza, entropía, etc.) investigando en qué medida las sociedades históricas han sido más o menos cuidadosas del ambiente. En este artículo el interés es abordar la temática del medio urbano porteño entre 1850 y 1915 desde otra perspectiva que se considera puede enriquecer el cuerpo actual de conocimientos sobre historia ambiental, profundizando especialmente sobre las concepciones científicas que por aquel entonces, guiaron la práctica profesional en materia de medio urbano.

Palabras clave

Medio ambiente urbano, Buenos Aires. Historia de las ideas científicas y profesionales sobre la ciudad.

\begin{abstract}
This article will approach the issue of urban Buenos Aires between 1850 and 1915 from a perspective that can enrich the existing body of knowledge on environmental history, especially deepening scientific conceptions that guided the practice in the field of urban environment.
\end{abstract}

\section{Key words}

Urban Environment, Buenos Aires. History of scientific and professional ideas about the city.

\section{Sumario}

Introducción

1.- La química, la higiene pública y la salubridad de las ciudades. Buenos Aires 18501890.

2.- Período 1890-1915. La higiene social y las nuevas nociones de ambiente. La ingeniería sanitaria y su concepción de medio urbano.

3.- Resumiendo

Referencias 


\section{Introducción}

En este artículo abordo la temática del medio ambiente urbano en Buenos Aires entre 1850 y 1915 , desde las siguientes dimensiones de análisis:

a. las profesiones que estudiaron y tutelaron el entorno urbano porteño en dicho período,

b. los problemas que signaron como prioritarios en distintos escenarios,

c. las teorías científicas y concepciones disciplinares que guiaron las prácticas sugeridas para la ciudad,

d. Ios términos utilizados a través del tiempo para denominar aquello que hoy llamamos medio ambiente urbano, señalando especialmente, la emergencia de palabras como medio, ambiente o medio ambiente en los textos relativos al campo urbano.

¿Por qué encarar la temática del medio ambiente urbano desde esta perspectiva de análisis?. Porque mayoritariamente, la bibliografía sobre historia ambiental e historia del ambiente producida en los últimos treinta y cinco años ha abordado la problemática desde la perspectiva ecológica y ecologista. Es decir, desde una mirada que toma categorías actuales del debate ambiental, investigando en cuanto los diferentes estilos de desarrollo de las sociedades históricamente consideradas, contribuyeron a forjar manejos más o menos cuidadosos del ambiente a lo largo de la historia.

A mi criterio, este tipo de "historia ecológica" resulta útil para responder a preocupaciones "actuales" del investigador ecologista, tales como evaluar la sostenibilidad del ambiente a lo largo del tiempo, pero en ocasiones cae en extrapolaciones conceptuales y en anacronismos al designar como "ecológicas" a medidas de ordenamiento urbano que fueron ideadas y puestas en práctica desde concepciones científicas y objetivos de intervención muy diferentes a los actuales, y muy lejanos a lo que hoy se entiende por "problemática ambiental".

Desde este lugar, mi hipótesis es que si bien la preocupación de las profesiones por la calidad del entorno urbano existió desde antaño en los profesionales de la ciudad, lo cierto es que los "problemas", las "ópticas científicas y disciplinares" y los "objetivos de intervención" que guiaron la práctica profesional por aquel entonces eran radicalmente diferentes a las actuales, y que resulta necesario rescatar la problemática desde su respectiva dimensión histórico temporal

De acuerdo con lo dicho, el objetivo del presente artículo es dar cuenta de las primeras profesiones que estudiaron los problemas del entorno urbano porteño entre 1850 y 1915, de las ideas científicas que guiaban la intervención profesional por aquel entonces, de los términos con que los profesionales "denominaron" aquello que hoy 
llamados medio ambiente cuando aún no existía como palabra consagrada, y en la misma línea, indicar la emergencia de la terminología "medioambiental" urbana en los textos ligados a la ciudad.

Desde este presupuesto de partida, y utilizando como eje de referencia la institucionalización de las distintas disciplinas que paulatinamente se ocuparon de la ciudad, es posible señalar dos grandes escenarios en los cuales diferentes profesiones se hicieron cargo de la problemática del entorno urbano porteño entre 1850 y 1915 :

(a) una primera etapa -1850 -1890 - en donde la química y la higiene pública constituyeron la voz autorizada en materia de ciudad, y

(b) un segundo período -1890 - 1915 -, en donde la higiene pública cede paso a la higiene social, al tiempo que va conformándose la ingeniería sanitaria, rama técnica que paulatinamente tomó en su seno las competencias que tradicionalmente habían sido abordadas por la higiene pública.

En este último es cuando lentamente se va institucionalizando un nuevo dominio de intervención: "el medio urbano".

Ahora bien, a continuación trataré de sintetizar los problemas, las concepciones científicas y las nociones claves utilizadas en materia de ambiente en cada uno de los períodos citados.

\section{1.- La química, la higiene pública y la salubridad de las ciudades. Buenos Aires 1850-1890}

En Buenos Aires la química fue una de las primeras ciencias en conformarse, debido a la presencia de profesionales extranjeros de prestigio tales como Miguel Puíggari o Charles Murray. En 1854 se forma la Asociación Farmacéutica y en 1858 comienza a aparecer la Revista Farmacéutica, en donde se publican sucesivos artículos relacionados con problemáticas de la ciudad, tales como los pozos artesianos, saladeros y mataderos y otros temas referidos a la calidad del aire, el agua y sol. En cuanto a los médicos, en 1854 se forma la Asociación Médica Bonaerense y en 1864 comienza a editarse la Revista Médico Quirúrgica, en donde aparecen gran cantidad de artículos relacionados con la ciudad.

La agenda de temas urbanos propuestos por estos profesionales abarcó desde los problemas ligados al "emplazamiento original de la ciudad" (localización geográfica, situación geológica, clima, vientos), hasta la "organización material" de la urbe, basada en principios higiénicos.

En los aspectos edilicios: Ancho de las calles, altura máxima de los edificios, relación entre altura y ancho, plazas y parques, y árboles en las aceras públicas. 
En lo relativo a servicios de higiene e infraestuctura: Limpieza pública, recolección y tratamiento de residuos, pavimentación, abastecimiento de agua y desagüe cloacal.

Por último, las localizaciones especiales para establecimientos insalubres: como la ubicación extra urbana de sitios signados como peligrosos o dañosos a la salud, básicamente los saladeros y mataderos, industrias, hospitales y cementerios fueron otra de sus preocupaciones.

La agenda de propuestas de esta etapa, anclaba en un conjunto de creencias epidemiológicas que hacían hincapié en las condiciones del "entorno" y la aparición de enfermedad. Estas teorías epidemiológicas, encuentran su génesis en la medicina hipocrática, en tanto fue Hipócrates quien formuló por primera vez la relación entre las condiciones climáticas, cambios estacionales y la "tipicidad" de la enfermedad que presentaba una región. Al decir de Urteaga, en "Naturaleza, Clima y Civilización"1 teoría epidemiológica, que ponía fuerte acento en la influencia del "ambiente" sobre la salud y la Teorías de los Climas (Siglo XVIII), que vinculaba las características físicas y morales de los hombres a las particularidades climáticas de los lugares que habitaban, constituyeron las primeras teorías sobre el ambiente aparecidas históricamente.

A partir del siglo XVIII, aquella antigua teoría "ambientalista" sobre la enfermedad, reformula sus postulados con los conocimientos de la química moderna, apareciendo formalmente con el nombre de neohipocratismo. Para esta teoría, la enfermedad no tiene aún origen microbiológico, sino que es asimilada a fermentaciones de orden "químico" producida por gases deletéreos (mortíferos) y sofocantes - no malsanos per se sino porque inhabilitan la actuación de otros gases benéficos como el oxígeno o el ozoe. Por la etapa, la materia orgánica en putrefacción: detritus de animales, basurales a cielo abierto, emanaciones de saladeros, mataderos, fábricas, gases exhalados de letrinas no sometidas a tratamiento, son señalados como la principal causa de producción de gases mortíferos conductores de enfermedad. En esta época, el aire, aún más que el agua, juega un rol esencial en la salud humana en tanto se lo considera el principal vector de enfermedades epidémicas._¿En qué se relacionan estas teorías sobre entorno y salud, con las propuestas urbanas?

Siguiendo a higienistas argentinos famosos como Eduardo Wilde o Guillermo Rawson existen ciertos "modificadores externos" que favorecen o alteran la salud poblacional. Algunos de estos modificadores son directamente naturales y dependen del emplazamiento original de la ciudad, tales como el clima o la humedad, y otros en cambio, están sujetos a la "organización material" de la urbe. Cuestiones como la calidad atmosférica, la temperatura ambiente, la mayor o menor cantidad de asoleamiento, la pureza del agua, son factores que inciden en la salud y que dependen exclusivamente del manejo que haga de ellos a nivel urbano. Para los químicos e

\footnotetext{
${ }^{1}$ URTEAGA, Luis; "Naturaleza, Clima y Civilización. Ideas Medioambientales en el siglo XVIII" en Historia de la Ciencia y de la Técnica N²7, Madrid, Akal, 1997.
} 
higienistas de este período, la adecuada "organización material" es la resultante del entrelazamiento específico entre ciertos elementos naturales y los aspectos construidos (organización material). Prácticas como la recolección diaria de residuos, la pavimentación, la limpieza de letrinas, la distribución de agua potable y el desagüe cloacal, la localización extraurbana de cementerios, saladeros e industrias, aminoran la actuación de los gases deletéreos, mientras que el ancho adecuado de calles, la relación entre altura y ancho de edificios, la creación de parques y plazas, y la arboleda urbana, favorecen la producción de gases benéficos, como el oxígeno o el ozoe.

Junto a estas teorías de orden epidemiológico, otras dos propuestas, resultan los elementos más significativos de las prácticas urbanas de este período: una es de orden económico y se vincula con los proyectos ligados a extraer beneficio económico de ciertas excreciones urbanas, como la basura o el líquido cloacal, y otro es de orden científico y está ligado al "principio de circulación constante de la materia", postulado que aún sigue vivo en ecología, y que ya aparece en los estudios de los higienistas y químicos de la etapa.

En cuanto a la faz económica, algunos ingenieros como Higgin, o profesionales como Aberg y empresarios interesados en obtener rentabilidad comercial, presentan sucesivos proyectos tendientes a fertilizar con abono humano campos especialmente designados para sembrar. Ello resolvía dos problemas de importancia: Uno sanitario, es decir, el destino final de las basuras o el desecho cloacal sin perjuicios ulteriores para la salud, y otro económico, en tanto se consideraba que tales "excreciones urbanas" servían para fertilizar la tierra produciendo cosechas altamente redituables en los mercados externos. Ligado a este criterio sanitario-económico, un postulado de mayor envergadura subayacía tras estas propuestas: "devolver a la tierra los principios fertilizantes que reclama", es decir el principio de "circulación permanente de la materia", estudiado por químicos extranjeros como Théodore de Saussure, Liebig, Boussingault $o$ Dumas y continuamente referenciados en Buenos Aires por profesionales locales como Miguel Puíggari, que fue junto a los ingenieros, naturalistas y empresarios antes citados, uno de los propulsores de la "irrigación de terrenos con fines agrícolas", que constituyó uno de los debates paradigmáticos de este período.

A mi criterio, estas medidas constituyeron el primer conjunto de intervenciones destinadas a mejorar la calidad del entorno urbano porteño. Sin embargo ellas no podrían ser calificadas como "ambientales" en el sentido estricto que hoy le damos al concepto. Constituyen prácticas de higiene urbana ideadas desde concepciones muy diferentes a los actuales y con objetivos de salubridad y salud pública, también muy distintos. Las terminologías utilizados por los higienistas de la etapa 1850-1890 para "nominar" lo que hoy llamamos "medio ambiente" fueron términos como "modificadores externos", "modificadores higiénicos", "modificadores externos de la salud", "circumfusa", "excreta", "ingesta", "aplicata", "miasma", "salubridad", "higiene". Por la etapa, términos como "medio o ambiente" están aún relegados al lenguaje físico químico y aún no son visualizables en los escritos referidos a la ciudad. 


\section{2.- Período 1890-1915. La higiene social y las nuevas nociones de ambiente. La ingeniería sanitaria y su concepción de medio urbano.}

Hacia 1890, aquella primera agenda de higiene pasará lentamente de "pública a social". ¿Cómo se resignifica la relación ambiente-ciudad, a partir de la emergencia del higienismo social?

Dos cambios fundamentales marcan la entrada en escena de la higiene social. Las transformaciones producidas por la revolución pasteuriana ${ }^{2}$ y la resignificación ideológica de las causas sociales que originan la enfermedad. En este sentido, la higiene social retoma el viejo cuerpo programático de la higiene pública: agua, recolección de residuos, pavimentos (causas directas) pero agrega otros ligados a las "causas indirectas": salarios, alojamiento, condiciones de trabajo de los obreros.

Con la "higiene social", viejos términos tradicionales del lenguaje urbano sanitario, tales como condiciones de higiene, salubridad, salubrificación, higiene pública, se reúnen con nuevas terminologías como "medio fabril", "temperatura ambiente", "medio social", "medio ambiente", "medio industrial", nuevos vocablos que se introducen con la medicina social, junto con otros como "medio físico" o "ambiente malsano".

Ellos designan viejos y nuevos temas del higienismo: calidad del aire, agua y sol, salubridad de la habitación, alojamiento popular, junto a nuevas temáticas específicas de finales del XIX, como higiene industrial, enfermedades profesionales, ruidos, automatismo, surmenage muscular o nervioso, seguros contra la invalidez y vejez, vivienda propia, y otro conjunto de expresiones que evocan nuevas y antiguas problemáticas de la higiene poblacional. Hacia fin de siglo, se institucionalizan los términos "medio", "ambiente" y "medio ambiente", abarcando campos temáticos que aluden a distintas dimensiones del ambiente: físico, natural y construido.

El "medio físico" abarca el estudio de los agentes naturales como el aire, el agua, el sol y calidad del suelo que son estudiados desde la perspectiva de las disciplinas tradicionales como la higiene y la química, y las ciencias nacientes como la bacteriología.

El medio construido pone el acento en la organización material del espacio en pos del mejoramiento de la salud y la calidad de vida. De acuerdo a los nuevos postulados de

\footnotetext{
2 La teoría microbiana de las enfermedades se construye entre 1865 y 1885 a través de distintas investigaciones con las cuales Pasteur se fue acercando a su objetivo final, es decir, la demostración de que la enfermedad está ligada a la existencia de gérmenes patógenos "específicos" que la provocan y no a miasmas existentes en cualquier elemento antihigiénico. Con estas investigaciones se revoluciona totalmente la antigua teoría sobre el origen de las enfermedades en tanto lo que comienza a investigarse es el germen "concreto" que provoca una enfermedad y la vacuna que lo combate, al tiempo que se renuevan absolutamente las medidas de profilaxia a partir de los nuevos conocimientos científicos.
} 
la microbiología y los nuevos problemas de fin de siglo, las temáticas centrales de esta etapa son: la "orientación" de las calles a fin de asegurar el asoleamiento mínimo diario de las viviendas, el alojamiento obrero, materiales higiénicos para la construcción de talleres industriales, la evacuación de líquidos cloacales y residuos domésticos tanto en el conventillo como en la industria, baños y duchas para la higiene de los obreros, temperatura adecuada en el trabajo. Todos problemas que encaró el higienismo social para mejorar la calidad del "ambiente" en que transita su vida del obrero, en la ciudad, en el trabajo o en la vivienda.

El "medio social" aparece tras este vasto campo de temas que no excluyen los infraestructurales, pero que se centran en la "calidad" de vida: la fatiga, el surmenage, la sobrecarga muscular o nerviosa, la dispersión que provoca el automatismo de ciertas tareas industriales, que constituyen las enfermedades profesionales típicas del "medio fabril". Junto con la tuberculosis, el alcoholismo o la prostitución, fueron parte de los padecimientos tratados por el higienismo social de fin de siglo que los vinculó al "ambiente malsano" que rodeaba la vida del trabajador: el alojamiento antihigiénico, la habitación hacinada, la carencia social y económica, el trabajo insalubre.

Como comenté anteriormente, con el higienismo social que emerge hacia 1890 se institucionalizan terminologías como "medio físico", "medio fabril", "ambiente malsano" o "medio ambiente". Ellas evocan diferentes dimensiones del ambiente que abarcan lo físico natural, lo construido y el ambiente social. Tres dimensiones detrás de las cuales se esconden tanto nuevas como antiguas problemáticas, a la vez que nuevas perspectivas científico ideológicas para abordarlas.

Paralelamente a la emergencia del higienismo social, otra profesión ligada al campo técnico se consolida lentamente y comienza a compartir las incumbencias y preocupaciones relativas al medio urbano: la "ingeniería sanitaria".

Tradicionalmente, los problemas técnicos relativos al saneamiento e higiene de la ciudad de Buenos Aires, fueron abordados por los ingenieros egresados de las primeras ramas formalizadas en Argentina: la ingeniería civil y la hidráulica. Los problemas centrales ligados a la calidad del entorno urbano que ocuparon a estos profesionales fueron: medios técnicos de abastecimiento de agua y desagüe cloacal, pavimentación: tipo de materiales a utilizar según suelo, clima, humedad, tránsito, etc, composición, tratamiento y disposición final de los residuos urbanos, además de la reutilización comercial de la basura, que constituye un debate central de los ingenieros hacia fin de siglo.

Retomando esta tradición disciplinar, hacia finales del siglo XIX, comienza a consolidarse lentamente la ingeniería sanitaria. Dicha rama técnica, fue conformándose lentamente al calor de varios factores que se articulan en paralelo. Los conocimientos adquiridos en las facultades, el contacto permanente con especialistas de otras profesiones, la práctica en las oficinas del estado, los viajes de estudio internacional, el trabajo dentro de las Comisiones de Salubridad y el vínculo constante con ingenieros extranjeros, fortalecieron y solidificaron el saber y la experiencia de los ingenieros 
locales. Hacia fin de siglo, la presión de los Congresos Internacionales - que reclaman la existencia de arquitectos e ingenieros sanitarios - y la relevancia que toma el agua, el tratamiento de residuos y la limpieza del subsuelo como elementos claves en la preservación de la salud pública a partir de la revolución microbiológica, serán factores esenciales en la consolidación de la técnica sanitaria como rama independiente.

A mi criterio, hacia 1915, la ingeniería sanitaria, cuyo campo de incumbencia será: el estudio de los "grandes factores salubres naturales: aire, luz, suelo, agua, captaje y distribución de aguas potables, evacuación y tratamiento de residuos, saneamiento", toma en su seno las competencias que tradicionalmente habían sido abordadas por la higiene pública, estableciéndose una división de funciones entre higienismo social y técnica sanitaria, en las que al ingeniero le corresponde la puesta a punto del "medio material".

Junto con el nacimiento de la ingeniería sanitaria se institucionalizan una serie de terminologías que aparecen constantemente en los escritos de los ingenieros sanitarios: "medio material", "medio artificial", "medio urbano". Ellos aluden a conceptos que aún siguen vigentes en la planificación urbano ambiental y también a términos que aún continúan utilizándose. ¿No se considera "medio artificial", al soporte tecnosférico edificado sobre un soporte natural?, ¿no es acaso el "medio material" la adaptación del hábitat construido a las necesidades de uso del "medio humano"?, ¿no es este acondicionamiento, en definitiva, el "medio urbano"?

Lo que quiero poner de manifiesto, es que hacia principios del siglo XX - y particularmente con la institucionalización de la ingeniería sanitaria - emergen terminologías y significados que están en las base de muchos de los actuales conceptos de la planificación urbano ambiental, aunque los "objetivos" que guían la práctica en una y otra etapa, son radicalmente diferentes, tal como se verá a continuación.

\section{3.- Resumiendo}

Entre 1850 y 1915 se institucionaliza lentamente un concepto: "el medio ambiente urbano". A mi criterio, su emergencia como nuevo objeto de problema, resume la historia de las disciplinas que sucesivamente se ocuparon del tema, las teorías usadas para encauzar la problemática en diferentes escenarios, la renovación de las teorías y la transferencia de conceptos entre las disciplinas, y un cuerpo de problemas alrededor de los cuales entre 1850 y 1915, fue paulatinamente consolidándose un nuevo dominio de intervención: "el medio ambiente urbano".

¿Pueden considerarse aquellas concepciones y prácticas sugeridas respecto al medio urbano similares a las actualmente sostenidas desde la ecología?

A mi parecer, varias de las nociones que ya aparecen entre el XIX y XX esencialmente con la emergencia de la ingeniería sanitaria - constituyen el antecedente directo de muchos conceptos actuales del campo ambiental, aunque lo 
cierto es que algunos criterios separan ampliamente aquellas concepciones y prácticas, de las actuales. Esencialmente el siguiente: en los albores del XX, el ambiente es definido "en pos de la salud pública": el sanitarismo, la preservación de la enfermedad, el acondicionamiento del hábitat construido en pos del confort humano, son los objetivos que guían la práctica de los profesionales de la ciudad.

Desde la década de 1970 en adelante, con la caída del ideal de progreso indefinido y el escepticismo en la "técnica" como instrumento superador de los obstáculos al desarrollo el "medio" y la "sostenibilidad del ambiente" pasan a ser los protagonistas. Cambio fundamental de perspectiva, objetivos y "objeto" de estudio, que sostenido en tradiciones y prácticas disciplinares anteriores se reformula para abordar nuevas problemáticas inexistentes hacia comienzos de siglo.

\section{Referencias}

\section{Bibliografía}

ALLEN, Adriana; 1996, Desarrollo Urbano Sustentable, Mar del Plata, Centro de Investigaciones Ambientales, FAUD, UNMdP.

BRAILOVSKY, Antonio y FOGUELMAN, Dina. 1993. Memoria Verde. Historia Ecológica de la Argentina., Buenos Aires, Sudamericana.

CARBONELL, Manuel; 1922, Evolución y Enseñanza de la Higiene, Buenos Aires, Rodríguez Giles.

CORBIN, Alain; 1987. El Perfume o el Miasma. El Olfato y lo Imaginario Social. Siglos XVIII al XX, México, Fondo de Cultura (1ra edición, Le miasme et la jonquille. L'Odorat et l'imaginaire social, París, Editions Aubier Montaigne, 1982).

DELEAGE, Jean Paul, Historia de la Ecología, Barcelona, Icaria, 1993, 364 págs. (1ra edición Histoire de l'ecologie, París, Editions La Découverte, 1991).

FERNANDEZ, Roberto; 1996, Teoría y Metodología de la Gestión Ambiental del Desarrollo Urbano, Mar del Plata, Centro de Investigaciones Ambientales -FAUDUNMdP, 1996 (2 volúmenes).

FERNANDEZ FIGUEROA, Enrique.1998. Problemas de Historia Urbana y Regional, Centro de Investigaciones Ambientales, FAUD, UNMdP.

GONZALEZ DE MOLINA, Manuel. 1993. Historia y Medio Ambiente, Madrid, Eudema.

TORRES, Horacio; "El Origen Interdisciplinario de los estudios urbanos" en Seminario Vaquerías'96, Buenos Aires, CRH-FADU-UBA. Vol 2. 


\section{REVISTA DE}

\section{URBANISMO}

ISSN 0717-5051

http://revistaurbanismo.vchile.cl

WILDE, Eduardo; 1878.Curso de Higiene Pública, Buenos Aires, Imprenta y Librería Mayo.

Nota: este escrito corresponde a una ponencia presentada a la CONFERENCIA INTERNACIONAL LA CULTURA ARQUITECTÓNICA HACIA 1900. REVALORIZACIÓN CRÍTICA Y PRESERVACIÓN PATRIMONIAL. ICOMOS Argentina - Gobierno de la Ciudad de Buenos Aires - Universidad "Torcuato Di Tella". Buenos Aires, 31 de agosto al 3 de septiembre de 1999. 